\section{Sicherheits-Broschüre}

\section{für den Umgang mit gefährlichen Stoffen}

Wenn Beschäftigte mit gefährlichen Stoffen arbeiten, brauchen sie einen ausreichenden Schutz. Dazu müssen Risiken erkannt und Maßnahmen ergriffen werden. Mit dem Einfachen Maßnahmenkonzept Gefahrstoffe (EMKG) hat die Bundesanstalt für Arbeitsschutz und Arbeitsmedizin (BAuA) eine neue praxisnahe Handlungshilfe entwickelt, die jetzt als Broschüre „BAuA-Handlungshilfe: Sicherheit bei der Arbeit mit Chemikalien herstellen" veröffentlicht worden ist. Mit dieser Anleitung lassen sich Gefährdungen beim Umgang mit gefährlichen Stoffen schnell und sicher erkennen und entsprechende Schutzmaßnahmen ableiten. Die Broschüre enthält die aktuellen Regelungen aus der Neufassung der Gefahrstoffverordnung von 2010 und die TRGS 400 "Gefährdungsbeurteilung für Tätigkeiten mit Gefahrstoffen". Zielgruppe des EMKG sind Verantwortliche in Klein- und Mittelständischen Betrieben, Sicherheitsfachkräfte, Betriebsärzte und überbetriebliche Beratungsdienste. Das Werk enthält leicht verständliche Informationen aus Sicherheitsdatenblättern und Betriebsbegehungen. Darüber hinaus ist die Broschüre dazu geeignet, die Wirksamkeit bereits getroffener Schutzmaßnahmen zu beurteilen.

Die Broschüre lässt sich unter www. baua.de/publikationen oder www.baua. de/de/Publikationen/Fachbeitraege/ Gd64.html herunterladen.

\title{
Trockeneisstrahlgerät reinigt große Kunststoffteile
}

Eür die Reinigung großer Kunststoff-

- flächen bietet die Firma Carvex das neue Trockeneisstrahlgerät Carvexblaster in drei Varianten an. Die Geräte zeichnen sich durch eine schonende, umweltfreundliche und gleichzeitig kraftvolle Reinigung aus. Sie eignen sich unter anderem für die Reinigung von Spritzgussformen, Formen zur PU-Fertigung oder die Vorbereitung für die Lackierung. Die drei Varianten unterscheiden sich zum einen durch die verschiedenen Betriebsüberdrücke (bis zu 10 beziehungsweise 15bar) und die Größe des Trockeneis-Vorratsbehälters. Die Variante Carvex-Blaster Automatik kann dank einer Roboterschnittstelle in vorhandene Anlagen integriert werden. Alle drei Varianten verfügen über ein robustes und einfach zu bedienendes Einschlauchsystem. Die Geräte arbeiten trotz ihrer überdurchschnittlichen Leistung

\section{Opferschicht erleichtert die Reinigung von Gitterrosten}

$D^{i}$ ie neue 2K-Gitterrostbeschichtung Feamtop 2901 von Ensutec Products erleichtert künftig das Entlacken von Gitterrosten. Das Produkt bildet eine Multiplex-Opferschicht. Es genügt, die Gitterroste einmal zu beschichten. Die Funktion der Oberfläche bleibt dennoch über einen langen Zeitraum erhalten. Wie der Hersteller betont, wird die Entlackung durch die Multiplex-Opferschicht deutlich erleichtert. Die Personal- und Reinigungskosten werden reduziert. In einem Anwendungsfall konnten die Entlackungskosten, die Transportkosten sowie die Kosten für Aus- und Einbau der Gitterroste um 80 Prozent gesenkt werden. Die langen Kabinenstillstandszeiten wurden erheblich verkürzt. Um die Funktionalität der Gitterroste zu erhalten, ist ein manuelles Abreinigen ausreichend.

Tel. 07371 96664-20, www.ensutec-products.de sparsam. Bei allen Geräten können die Parameter "Strahldruck" und "Menge der Trockeneispellets" getrennt voneinander eingestellt werden. Die Pelletgröße wird über einen Splitter reguliert.

Tel. 02635 789-0, www.carvex.de

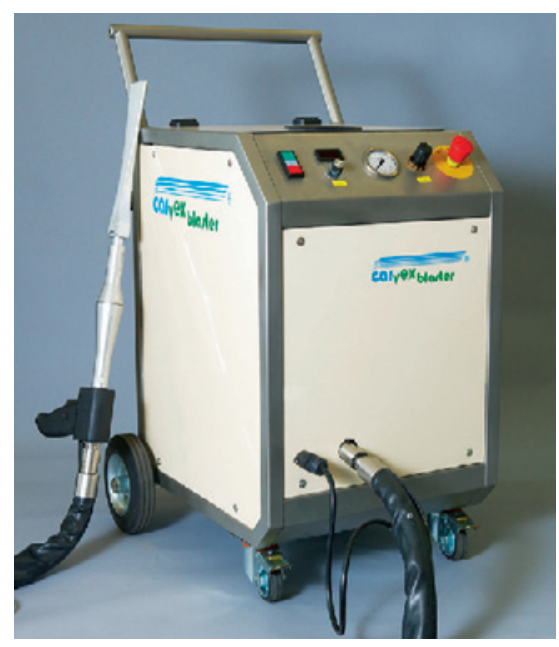

\section{Feinste Partikel aus Schweiß- und Schneidabluft filtern}

Eür die Filterung von feinem, schwer handzuhabendem Staub und Rauch aus Schweiß-, Schneid- und Beschichtungsprozessen hat die Freudenberg Filtration Technologies die Viledon sinTexx-Plus-Filterpatronen auf den Markt gebracht. Die Patronen sind mit einer Nanofaserauflage ausgestattet, die sehr feine Partikel aus der abgesaugten Abluft entfernt. Damit wird eine höhere Abscheideleistung erreicht als bei konventionellem, rilliertem Polyester-Material oder bei ePTFE-Membranen. Arbeitsplatzgrenzwerte lassen sich so leicht einhalten. Darüber hinaus wird die höhere Abscheideleistung schon am Anfang erreicht. Die neuen Filter haben von Anfang an eine höhere Abscheideleistung. Dank der Nanofaserauflagen haben die Filterpatronen einen geringeren Durchströmungswiderstand. Damit verringern sich der Druckluft- und Stromverbrauch deutlich.

Tel. 06201 80-0, www.freudenberg-filter.com 